\title{
Hormonal and Sex Impact on the Epidemiology of Canine Lymphoma
}

\author{
J. Armando Villamil, ${ }^{1}$ Carolyn J. Henry, ${ }^{1,2}$ Allen W. Hahn, ${ }^{1}$ Jeffrey N. Bryan, ${ }^{3}$ \\ Jeff W. Tyler, ${ }^{1,4}$ and Charles W. Caldwell ${ }^{5}$
}

${ }^{1}$ Department of Veterinary Medicine and Surgery, University of Missouri, Columbia, MO 65211, USA

${ }^{2}$ Hematology/Oncology Division, Department of Internal Medicine, University of Missouri, Columbia, MO 65211, USA

${ }^{3}$ Department of Veterinary Clinical Sciences, Washington State University, Pullman, WA 99164, USA

${ }^{4}$ Department of Veterinary Medicine and Surgery, University of Missouri Public Health Program, University of Missouri, Columbia, MO 65211, USA

${ }^{5}$ Department of Pathology and Anatomical Sciences, School of Medicine and Ellis Fischel Cancer Center, University of Missouri, Columbia, MO 65211, USA

Correspondence should be addressed to J. Armando Villamil, villamilj@missouri.edu

Received 5 May 2009; Revised 27 August 2009; Accepted 9 December 2009

Recommended by Paolo Boffetta

\begin{abstract}
The Surveillance Epidemiology and End Results data demonstrate that the risk of non-Hodgkin lymphoma is lower for women, but that the incidence increases after fifty years of age, at which menopause is regularly reached, suggesting that female hormones may be protective for NHL. This study examines the influence of sex on lymphoma risk in a relevant large animal model. Records for dogs in the Veterinary Medical Database were analyzed from 1964 to 2002 . Risk ratios were calculated to evaluate associations between sex, neutering status, and lymphoma occurrence. A total of 14,573 cases and 1,157,342 controls were identified. Intact females had a significantly lower risk of developing lymphoma, Odds Ratio $0.69(0.63-0.74)$ with a $P<.001$. We conclude that there is a sex effect on NHL risk in dogs similar to humans. We hypothesize that the hormone levels of intact females lower the risk of NHL. The possibility of a protective role of endogenous estrogens in the etiology of NHL should be investigated.
\end{abstract}

Copyright ( $) 2009$ J. Armando Villamil et al. This is an open access article distributed under the Creative Commons Attribution License, which permits unrestricted use, distribution, and reproduction in any medium, provided the original work is properly cited.

\section{Introduction}

Women are known to have a lower incidence of nonHodgkin lymphoma (NHL) than men (15.8 cases/100,000 person-years (py) versus 23.2/ 100,000 py from 1998 to 2002) [1]. The observed difference has persisted over the past two decades. Although the exact mechanism and cause of the disparity is unknown, the interaction of hormones and estrogen or progesterone receptors on gene expression has been postulated as one logical mechanism. The finding of specific polymorphisms in the estrogen receptor 1 associated with higher susceptibility to lymphoma supports a hormonal role in carcinogenesis [2].

Naturally occurring lymphoma is a common malignancy in dogs. The NHL classification schemes used in human medicine have been adapted for use in dogs [3]. Investigators have suggested that companion dogs can serve as an important comparative model $[4,5]$. The disease occurs spontaneously in companion dogs with genetic, epigenetic, histologic, therapeutic, and natural history similarities with humans. The shorter lifespan of dogs compresses progression time and permits more rapid study of disease epidemiology and biological behavior [6]. Finally, dogs are exposed to similar environmental and hormonal factors as humans and may serve as an environmental sentinel and model of prevention of NHL [7].

Hormonal influence on the causation, prevention, and treatment has been well documented in the etiology of several canine tumors. There is an established relationship between the incidence of canine mammary tumors and the timing of ovariectomy. Dogs spayed before their first heat cycle are significantly less likely to develop mammary tumors 
[8]. Perianal adenomas are considered to be androgen dependent, occurring primarily in male intact dogs and remitting following castration [9]. Conversely, neutered male dogs have a significantly increased risk for all prostate cancers when compared to their intact counterparts [10].

The etiologic influence of hormone status on the risk of development of NHL has been examined in human oncology. In a study of 37,220 Iowa women ages 55 to 69 years, current users of hormonal replacement therapy were at increased risk for NHL [11]. Risk factors including endogenous sex hormones, age at menarche, age at menopause, type of menopause, history of infertility, number of miscarriages, history of induced abortion, number of livebirths, and age at first livebirth were evaluated and were not strongly related to NHL incidence. A second study found an inverse association with the number of breast-fed children, with incidence for NHL decreasing after two children [12]. In the evaluation of hormonal effects on cancer development in the human population, only females routinely experience dramatic changes in sex hormone levels over their lifetimes. Available information on similar effects in the male population is extremely limited. The dog population contains large numbers of both intact and neutered individuals, allowing the altering of endogenous hormone status to be evaluated in a large epidemiologic study.

The Veterinary Medical Database (VMDB) is the most comprehensive database in veterinary medicine. The VMDB contains individual patient records from 27 North American veterinary teaching hospitals, including more than 6 million records of various animal species from 1964 to present. The VMDB data permits comparisons between hormonally intact and neutered dogs in both sex groups with a diagnosis of canine lymphoma.

Canine lymphoma has historically been thought to be more prevalent in males compared to females, but this was based largely upon anecdotal information. An article published by Dorn in 1970 reported that there was no difference between sexes in the risk of developing lymphoma; [13] to date no other study has evaluated this specific relationship. The purpose of this study was to examine records from the VMDB to evaluate the relationship between sex and lymphoma risk in dogs, with the hypothesis that intact female dogs are at lower risk for NHL.

\section{Materials and Methods}

The VMDB was queried to identify all canine records entered between 1964 and 2002. (VMDB's URL http://www.vmdb.org/ VMDB/CERF, PO Box 3007, 1717 Philo Rd, Suite 15,Urbana, IL 61803-3007. VMDB, Inc. does not make any implicit or explicit opinion on the subject of this study.) Cases were defined as dogs with lymphoma, including lymphoma type not specified (morphology code 8301), giant follicular lymphoma (morphology codes 8303 or 8330), or lymphosarcoma (morphology code 8310) according to the Standard Nomenclature of Veterinary Diseases and Operations (SNVDO). The control population was defined as all dogs with any diagnosis other than the 4 previously specified lymphoma diagnoses that never were reported to have lymphoma. Because each office visit or hospital stay results in the production of a unique entry in the VMDB, individual dogs may enter the database multiple times during their lifetime. Consequently, only a single, randomly selected record was used for each individual dog in the control population. For dogs with lymphoma, we used data from the first recorded visit in which the diagnosis of lymphoma was listed. To ensure that each dog was represented only once in the dataset, the data was sorted by the patient identification number and then filtered using SAS statistical software ( SAS for Windows, version 9.13, SAS Institute Inc, Cary, NC). Duplicate records were identified and deleted.

Variables extracted from records included sex (intact female, spayed female, intact male, neutered male), age, diagnosis code, visit year, and breed code. Age categories included eight age ranges as recorded in the VMDB (2-6 months, 6-12 months, $1-2$ years, $2-4$ years, $4-7$ years, $7-10$ years, $10-15$ years or $>15$ years). Due to the low incidence of lymphoma in dogs less than 4 years of age, the 2-6 months, 6-12 months, 1-2 years, and 2-4 years categories were combined into a less than 4-year category. The records of control dogs less than 2 months of age were deleted from the dataset because no lymphoma diagnoses were noted in this age category. All records with unknown age, sex, breed, visit year, or diagnosis codes were excluded.

Given the potential for confounding effects of breed, and age, a paired analysis was performed for risk estimates by sex. In this analysis, a control record was selected for each case record. Control records were matched first on age and then on breed. Due to the volume of records within the VMDB, it was possible to match all controls and cases exactly for both age and breed. When multiple matched controls were available for a single case record, one control was randomly selected. For each sex category a $2 \times 2$ table was generated, based on the lymphoma status in each pair. In these tables, cell A included paired data when both the case and control members were of the sex under consideration, $\mathrm{B}$ when the case was a member of the sex under consideration and the control was not, $\mathrm{C}$ when the control was a member of the sex category under consideration and the case was not, and D when neither the case nor control member of the pair was a member of the sex under consideration. Using matched data, the odds ratio (OR) for lymphoma by specific sex category was determined using as previously described for matched data [14]. Thus, the risk of lymphoma for each sex group was calculated compared to the matched case-control population.

In addition, logistic regression models were developed using all the cases and controls available in the VMDB to predict the probability of the diagnosis of lymphoma as a function of sex, breed, and age. Only breeds which had a significance $(P<.001)$ on a univariate analysis were considered; all breeds without significance were grouped as "other pure breeds" for the multivariate analysis. Regression coefficients were used to calculate the odds ratio with $95 \%$ confidence intervals for each breed, estimating the risk of the diagnosis of lymphoma relative to the mixed breed 
TABLE 1: Sex distribution of lymphoma dogs evaluated in the VMDB (1964-2002).

\begin{tabular}{lcc}
\hline Sex & Case (\%) & Control (\%) \\
\hline All Females & $6,936(47.6)$ & $582,941(50.4)$ \\
All Males & $7,637(52.4)$ & $574,401(49.6)$ \\
Female Intact & $1,704(11.7)$ & $301,208(26.0)$ \\
Female Spayed & $5,232(35.9)$ & $281,733(24.4)$ \\
Male Intact & $4,437(30.4)$ & $435,313(37.6)$ \\
Male Castrated & $3,200(22.0)$ & $139,088(12.0)$ \\
\hline
\end{tabular}

TABle 2: Odds Ratio and 95\% Confidence Intervals for the paired (breed and age) data for Canine Lymphoma in the VMDB (19642002).

\begin{tabular}{lcc}
\hline Sex & Odds ratio & 95\% confidence interval \\
\hline Intact Female & 0.69 & $0.63,0.74$ \\
Spayed female & 1.02 & $0.96,1.08$ \\
Intact Male & 1.32 & $1.24,1.41$ \\
Neutered Male & 0.91 & $0.85,0.97$ \\
\hline
\end{tabular}

population. Only breeds with significant difference in the multivariate analysis are presented in the results table.

Comparative data for human NHL were gathered from the SEER website. ( SEER Cancer Statistics Review, 1975-2003, National Cancer Institute. Bethesda, MD, http://seer.cancer.gov/csr/1975_2003/, based on November 2005 SEER data submission, posted to the SEER website, 2006. The U. S. Government, National Cancer Institute, and their employees do not make any implicit or implied opinion on the subject of this paper.) Utilizing the fast stats tool, a query of age-adjusted rates for the incidence of NHL was performed from 1975 to 2005 for all races. Age was limited to $<50$ years for Figure 1 and $>50$ years for Figure 3 (data downloaded on January 20, 2009).

\section{Results}

A total of 1,171,915 individual canine records were present in the VMDB from 1964 to 2002; 1,157,342 constituted the control population and 14,573 the case population. A diagnosis of lymphoma is listed in $1.2 \%$ of the total accessions in the VMDB. Sex distribution of lymphoma dogs evaluated at North American veterinary teaching hospitals from 1964 to 2002 is presented in Table 1. Age distribution is presented in Table 3 .

To evaluate the effect of sex on lymphoma over those 38 years, 14,573 case-control pairs were identified based on age and breed. Table 2 presents the odds ratios and 95\% confidence interval for the development of lymphoma for each specific sex category. All intervals are mutually exclusive from each other. In the Logistic regression performed using the unmatched data, the female intact category was also the least likely to develop lymphoma.

The incidence of human NHL for all races in the population $<50$ years of age according to sex was extracted from the SEER database (Figure 1). It is apparent that females have a lower risk of developing NHL when compared to males. This difference is consistent throughout the time span under consideration. Figure 2 presents all intact dogs with the diagnosis of lymphoma out of the total population by year. The rate of a lymphoma diagnosis is lower in female dogs for every year examined. For humans over 50 years of age (Figure 3 ), the incidence difference between males and females appears decreased. A similar decrease in the difference between the rates appears for the altered canine population (Figure 4).

Overall, a decreased risk of developing NHL is noted in females of both the human and canine population. This decrease is less apparent after 50 years of age in humans or after sex alteration is performed in the canine population (Figures 3 and 4).

Dogs of less than 4 years of age are less likely to develop lymphoma compared to the rest of the population (Table 3 ). The most commonly represented breeds in the lymphoma group were mixed breed $(n=3,197)$, Golden retriever $(n=1,474)$, Labrador retriever $(n=854)$, German shepherd $(n=733)$, and Boxer $(n=677)$. When the odds ratio for lymphoma was examined in those breeds with more than one thousand control dogs, Bullmastiff, Boxer, Bernese mountain dog, Scottish terrier and Golden setters had the highest risk of lymphoma (Table 4). These breed predispositions may represent heritable factors contributing to increased frequency in these breeds. Elucidation of such genetic factors may help identify risk within human families or ethnic groups at higher risk for developing NHL.

\section{Discussion}

To the authors' knowledge, this is the only specific epidemiologic evaluation of sex risk for canine lymphoma. The VMDB is one of the most comprehensive databases in veterinary medicine, containing more than six million visit records. The VMDB was queried only to 2002 because after that year, the enrollment of cases was less consistent. With an extensive number of cases from multiple veterinary teaching hospitals across North America, geographical bias has likely been minimized. Age and breed bias must also be considered. To address this bias, we paired each individual lymphoma case with a randomly picked control of identical age category and breed variable. One of the major weaknesses of the data collected from the VMDB is possible misclassification error when data is entered; however, this should be random and eliminated with the design used in this study.

We conclude that the epidemiologic data from the VMDB supports a similar decreased risk for intact female dogs, as for women who have a lower risk than men of developing lymphoma. This sex effect has been consistent over the past few decades for both species. An interesting difference is noted for the altered dog and aged human population, where the difference in incidence of lymphoma in males and females becomes significantly smaller. We theorize that this difference may be attributed to the hormonal changes that occur once dogs are sexually altered via ovariohysterectomy (spay), similar to when women reach 
TABLE 3: Logistic regression model defining the risk of a diagnosis of lymphoma in dogs in the VMDB (1964-2002) as a function of age.

\begin{tabular}{|c|c|c|c|c|c|}
\hline Age Group & Case (\%) & Control (\%) & Odds Ratio & 95\% C.I. & $P$-value \\
\hline Less than 4 years & $1,791(12.3)$ & $606,667(52.4)$ & 1 & $*$ & $*$ \\
\hline $4-7$ years & $4,626(31.7)$ & $222,206(19.2)$ & 6.73 & $(6.36,7.11)$ & $<.0001$ \\
\hline $7-10$ years & $4,385(30.1)$ & $173,877(15.0)$ & 7.78 & $(7.35,8.24)$ & $<.0001$ \\
\hline $10-15$ years & $3,553(24.4)$ & $142,778(12.4)$ & 8.06 & $(7.60,8.55)$ & $<.0001$ \\
\hline More than 15 years & $218(1.5)$ & $11,814(1.0)$ & 6.96 & $(6.03,8.03)$ & $<.0001$ \\
\hline
\end{tabular}

* Baseline exposure; therefore coefficient and $P$ values are not provided and OR is defined as 1.

TABLE 4: Logistic regression model defining the risk of a diagnosis of lymphoma in dogs in the VMDB (1964-2002) as a function of breed.

\begin{tabular}{|c|c|c|c|c|c|}
\hline Breed & Case & Control & Odds Ratio & 95\% C.I. & $\mathrm{P}$ value \\
\hline Mix Breed & 3140 & 270503 & 1 & * & $*$ \\
\hline Bullmastiff & 51 & 1399 & 4.83 & $(3.62,6.44)$ & $<.0001$ \\
\hline Boxer & 675 & 14541 & 4.05 & $(3.71,4.41)$ & $<.0001$ \\
\hline Bernese Mountain Dog & 43 & 1301 & 3.64 & $(2.66,4.96)$ & $<.0001$ \\
\hline Scottish Terrier & 261 & 5647 & 3.39 & $(2.98,3.86)$ & $<.0001$ \\
\hline Gordon Setter & 50 & 1503 & 3.31 & $(2.48,4.41)$ & $<.0001$ \\
\hline Irish Wolfhound & 60 & 2135 & 3.23 & $(2.48,4.20)$ & $<.0001$ \\
\hline Basset Hound & 358 & 9945 & 3.22 & $(2.88,3.60)$ & $<.0001$ \\
\hline Golden Retriever & 1462 & 41705 & 3.12 & $(2.93,3.32)$ & $<.0001$ \\
\hline Rottweiler & 381 & 17174 & 2.71 & $(2.43,3.02)$ & $<.0001$ \\
\hline Vizsla & 55 & 2041 & 2.66 & $(2.02,3.49)$ & $<.0001$ \\
\hline Bouvier des Flandres & 42 & 1510 & 2.66 & $(1.94,3.63)$ & $<.0001$ \\
\hline Old English Sheepdog & 158 & 5988 & 2.48 & $(2.11,2.92)$ & $<.0001$ \\
\hline Saint Bernard & 176 & 9728 & 2.34 & $(2.01,2.74)$ & $<.0001$ \\
\hline Pembroke Welsh Corgi & 47 & 1845 & 2.08 & $(1.55,2.79)$ & $<.0001$ \\
\hline Airedale Terrier & 113 & 4778 & 1.92 & $(1.58,2.32)$ & $<.0001$ \\
\hline Samoyed & 45 & 7199 & 0.55 & $(0.41,0.74)$ & $<.0001$ \\
\hline Brittany Spaniel & 48 & 9116 & 0.48 & $(0.36,0.64)$ & $<.0001$ \\
\hline Maltese & 27 & 5240 & 0.42 & $(0.29,0.62)$ & $<.0001$ \\
\hline Dutch Pug & 24 & 5960 & 0.39 & $(0.26,0.58)$ & $<.0001$ \\
\hline Yorkshire Terrier & 57 & 13549 & 0.35 & $(0.27,0.45)$ & $<.0001$ \\
\hline Poodle, Miniature & 166 & 37939 & 0.29 & $(0.25,0.34)$ & $<.0001$ \\
\hline Pomeranian & 27 & 8982 & 0.28 & $(0.19,0.41)$ & $<.0001$ \\
\hline Chihuahua & 37 & 12522 & 0.28 & $(0.20,0.38)$ & $<.0001$ \\
\hline Poodle, Toy & 46 & 17326 & 0.20 & $(0.15,0.27)$ & $<.0001$ \\
\hline Dachshund & 68 & 26540 & 0.18 & $(0.15,0.22)$ & $<.0001$ \\
\hline Pekingese & 21 & 10389 & 0.18 & $(0.12,0.28)$ & $<.0001$ \\
\hline
\end{tabular}

*Baseline exposure; therefore coefficient and $P$ values are not provided and OR is defined as 1.

menopause. Due to the paucity of hormonally altered men, we cannot compare the effect of hormonal differences on lymphoma risk in the male population. Dogs are commonly neutered and hormonal changes are documented to occur. Thus, the canine model provides a unique resource for the study of hormonal influence on male NHL risk. Women with a history of oophorectomy had a very slight elevation in risk compared to women with no such history [12]. Since oophorectomy is not commonly performed, it is difficult to compare this practice to spaying in dogs. Therefore for this study we have assumed that hormonal changes that occur in women once they reach menopause are similar to those that occur in female dogs when they are altered. The elucidation of these hormonal changes after menopause in humans or alteration in dogs bears further investigation.

One of the limitations of this study is the lack of canine lymphoma subtype classification present in the VMDB. In dogs, the clinical diagnosis of lymphoma is usually limited to cell morphology, immunophenotype (CD3 for T cell, CD79a for $B$ cell), and assessment of nodal architectural [15]. Indolent lymphomas, which are similar to low-grade lymphomas of humans, are rarely recognized. The types of indolent lymphoma reported in dogs include mantle cell, marginal 


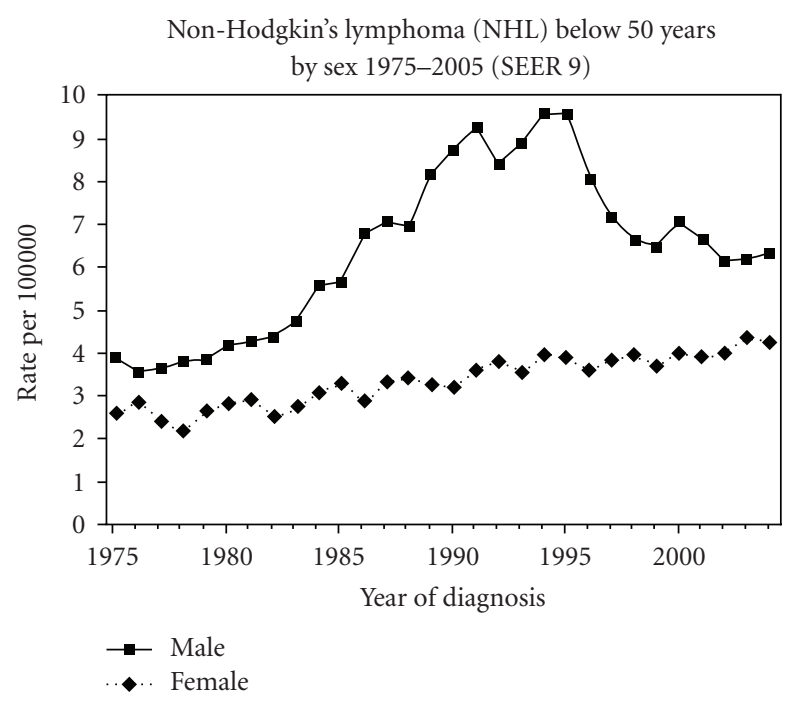

FIgURE 1: Incidence for all races of human NHL in individuals less than 50 years of age, adapted from the SEER webpage (http://www.seer.cancer.gov/). Rates are adjusted to the 2000 US Std. Population (19 age groups Census P25-1130).

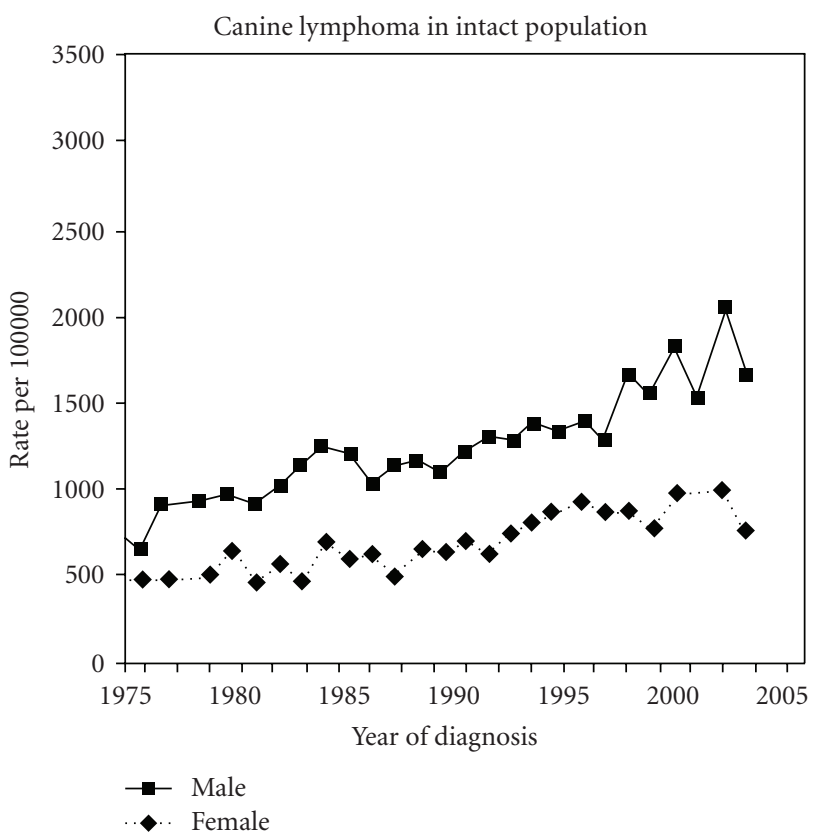

FIGURE 2: Rate of lymphoma in the intact canine population in the VMDB. Dashed line represents female intact (FI) dogs and solid line represents male intact (MI) dogs.

zone, follicular, and T-zone lymphomas [16]. Recent studies suggest a genetic factor in the etiology of lymphoma due to the breed association with immunophenotype and possibly histological subtypes $[17,18]$. In the human population, NHL is known to be a complex and heterogeneous set of lymphoma subtypes [19]. Subtypes include diffuse large B-cell lymphoma (DLBCL) and follicular lymphoma (FL).

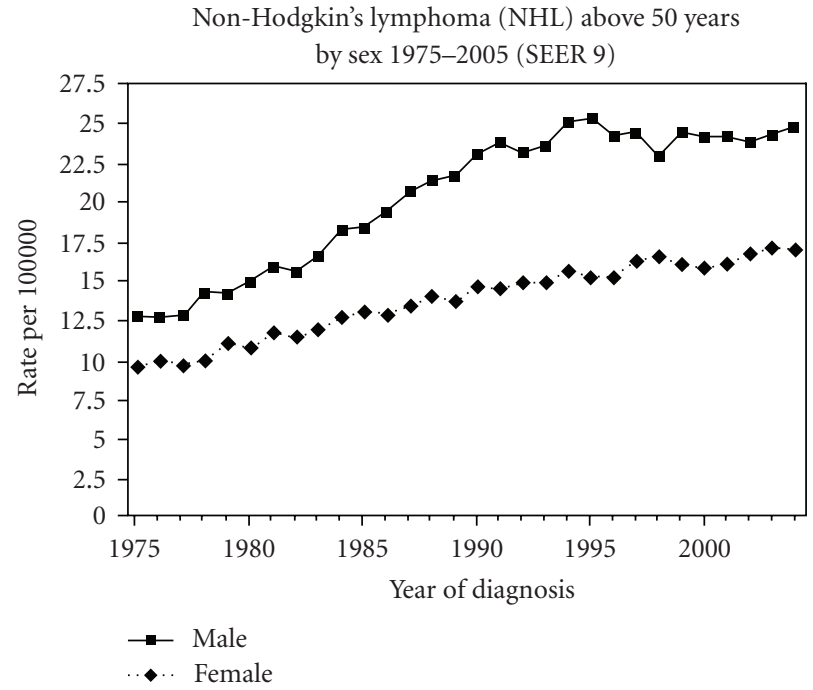

FIGURE 3: Incidence for all races of human NHL in individuals greater than 50 years of age, adapted from the SEER webpage (http://www.seer.cancer.gov/). Rates are adjusted to the 2000 US Std. Population (19 age groups Census P25-1130).

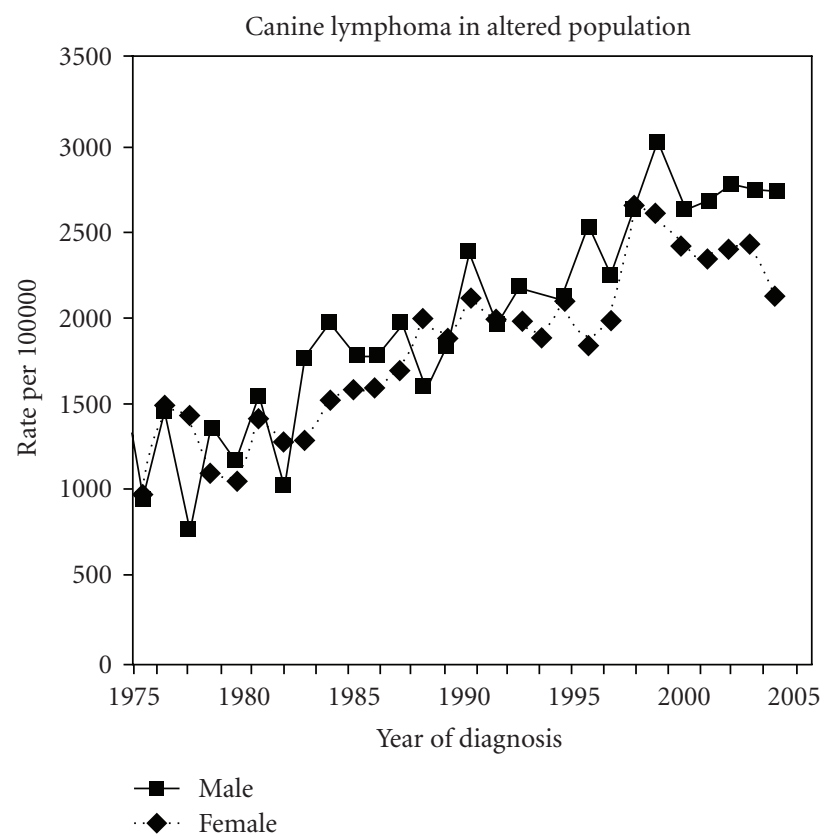

FIGURE 4: Rate of lymphoma in the canine altered population in the VMDB. Dashed line represents female spayed (FS) dogs and solid line represents male neutered $(\mathrm{MN})$ dogs.

Estrogen and other reproductive hormones may affect the risk of NHL subtypes such as DLBCL [20]. In addition, genetic variation and a wide variety of receptor haplotypes have been identified in human medicine. This variability may affect the risk and outcome of canine lymphoma and human NHL patients. More detailed prospective studies should be performed to evaluate these differences. 
When the multivariate logistic regression analysis was performed, sex, age, and breed categories showed significance in the development of lymphoma. The category of $7-$ 10 years of age appears to be predisposed while less than 4 years of age seems to be less likely to develop lymphoma. Among breeds Bullmastiff, Boxers, Bernese Mountain Dog and Scottish terriers showed an increased risk of lymphoma. Dachshund and Pekingese were less frequent than background. These findings are consistent with previously published data and may represent genetic susceptibility or protection [21].

Over the last decade, the relevance of veterinary comparative oncology to pharmaceutical and human medical oncology research has been recognized. The movement toward utilizing canine models in human medical research is primarily due the previously mentioned strengths of the canine model and the similarities between dogs and humans. In addition, the Comparative Oncology Trials Consortium (COTC) has formalized efforts to use companion dogs in human research by bringing together various institutions to work under the umbrella institution of the National Cancer Institute. The advantages of these efforts include rapid case accrual, decrease in the geographical distribution bias, and minimization of operational bias. More recently the creation of the Canine Comparative Oncology and Genomics Consortium (CCOGC) has opened the door for future studies of comparative animal models, accessible via a centralized biospecimen repository.

In conclusion, human NHL and canine lymphomas are similar diseases, making the dog an attractive model for this comparative evaluation. Our data emphasizes the similar correlation between presumptive hormone status and development of lymphoma in both species. Results of this analysis provide justification for further investigation of the hormonal influence on the risk of developing lymphoma.

\section{Acknowledgments}

The authors would like to acknowledge the partial support provided by Minority Bio-Medical Researchers Training Initiative (MBRTI) MU/NIH Science Education Partnership Awards (Villamil), University of Missouri-Columbia, the support from the National Library of Medicine Biomedical and Health Informatics Research training Grant 2-T15LM07089 (Bryan), National Cancer Institute Grants CA 100055 and CA 097880 and the CRC Missouri Chair in Cancer Research (Caldwell), and the Tom and Betty Scott Endowed Program in Veterinary Oncology University of Missouri (Villamil, Henry).

\section{References}

[1] D. D. Alexander, P. J. Mink, H. O. Adami, et al., "The non-Hodgkin lymphomas: a review of the epidemiologic literature," International Journal of Cancer, vol. 120, pp. 1-39, 2007.

[2] C. F. Skibola, P. M. Bracci, E. Halperin, et al., "Polymorphisms in the estrogen receptor 1 and vitamin $\mathrm{C}$ and matrix metal- loproteinase gene families are associated with susceptibility to lymphoma," PLoS ONE, vol. 3, no. 7, article e2816, 2008.

[3] C. Fournel-Fleury, J. P. Magnol, P. Bricaire, et al., "Cytohistological and immunological classification of canine malignant lymphomas: comparison with human non-hodgkin's lymphomas," Journal of Comparative Pathology, vol. 117, no. 1, pp. 35-59, 1997.

[4] D. M. Vail and E. G. MacEwen, "Spontaneously occurring tumors of companion animals as models for human cancer," Cancer Investigation, vol. 18, no. 8, pp. 781-792, 2000.

[5] C. Khanna, K. Lindblad-Toh, D. Vail, et al., "The dog as a cancer model," Nature Biotechnology, vol. 24, no. 9, pp. 10651066, 2006.

[6] M. C. Paoloni and C. Khanna, "Comparative oncology today," Veterinary Clinics of North America: Small Animal Practice, vol. 37, no. 6, pp. 1023-1032, 2007.

[7] L. B. Bronden, A. Flagstad, and A. T. Kristensen, "Veterinary cancer registries in companion animal cancer: a review," Veterinary and Comparative Oncology, vol. 5, no. 3, pp. 133144, 2007.

[8] R. Schneider, C. R. Dorn, and D. O. Taylor, "Factors influencing canine mammary tumor development and post surgical survival," Journal of the National Cancer Institute, vol. 43, 1969.

[9] G. P. Wilson and H. M. Hayes Jr., "Castration for treatment of perianal gland neoplasms in the dog," Journal of the American Veterinary Medical Association, vol. 174, pp. 1301-1303, 1979.

[10] J. N. Bryan, M. R. Keeler, C. J. Henry, et al., "A population study of neutering status as a risk factor for canine prostate cancer," Prostate, vol. 67, no. 11, pp. 1174-1181, 2007.

[11] J. R. Cerhan, C. M. Vachon, T. M. Habermann, et al., "Hormone replacement therapy and risk of non-Hodgkin lymphoma and chronic lymphocytic leukemia," Cancer Epidemiology Biomarkers and Prevention, vol. 11, no. 11, pp. 1466-1471, 2002.

[12] J. R. Cerhan, T. M. Habermann, C. M. Vachon, et al., "Menstrual and reproductive factors and risk of non-Hodgkin lymphoma: the Iowa women's health study (United States)," Cancer Causes \& Control, vol. 13, pp. 131-136, 2002.

[13] C. R. Dorn, D. O. Taylor, and R. Schneider, "The epidemiology of canine leukemia and lymphoma," The Bible of Haematology, pp. 403-415, 1970.

[14] N. Mantel and W. Haenszel, "Statistical aspects of the analysis of data from retrospective studies of disease," Journal of the National Cancer Institute, vol. 22, pp. 719-748, 1959.

[15] P. G. Greenlee, D. A. Filippa, F. W. Quimby, et al., "Lymphomas in dogs. A morphologic, immunologic, and clinical study," Cancer, vol. 66, no. 3, pp. 480-490, 1990.

[16] V. E. Valli, W. Vernau, L. P. de Lorimier, et al., "Canine indolent nodular lymphoma," Veterinary Pathology, vol. 43, no. 3, pp. 241-256, 2006.

[17] J. F. Modiano, M. Breen, R. C. Burnett, et al., "Distinct B-cell and T-cell lymphoproliferative disease prevalence among dog breeds indicates heritable risk," Cancer Research, vol. 65, no. 13, pp. 5654-5661, 2005.

[18] M. Pastor, K. Chalvet-Monfray, T. Marchal, et al., "Genetic and environmental risk indicators in canine non-Hodgkin's lymphomas: breed associations and geographic distribution of 608 cases diagnosed throughout France over 1 year," Journal of Veterinary Internal Medicine, vol. 23, no. 2, pp. 301-310, 2009.

[19] J. S. Lee, P. M. Bracci, and E. A. Holly, "Non-Hodgkin lymphoma in women: reproductive factors and exogenous hormone use," American Journal of Epidemiology, vol. 168, no. 3, pp. 278-288, 2008. 
[20] Y. Zhang, T. R. Holford, B. Leaderer, et al., "Menstrual and reproductive factors and risk of non-Hodgkin's lymphoma among connecticut women," American Journal of Epidemiology, vol. 160, no. 8, pp. 766-773, 2004.

[21] S. Withrow and D. Vail, Withrow and MacEwen's Small Animal Clinical Oncology, Saunders Elsevier, Saint-Louis, Mo, USA, 2007. 


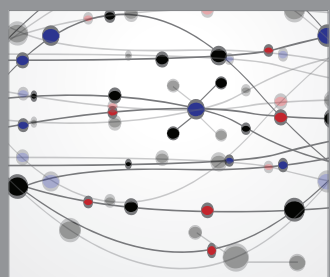

The Scientific World Journal
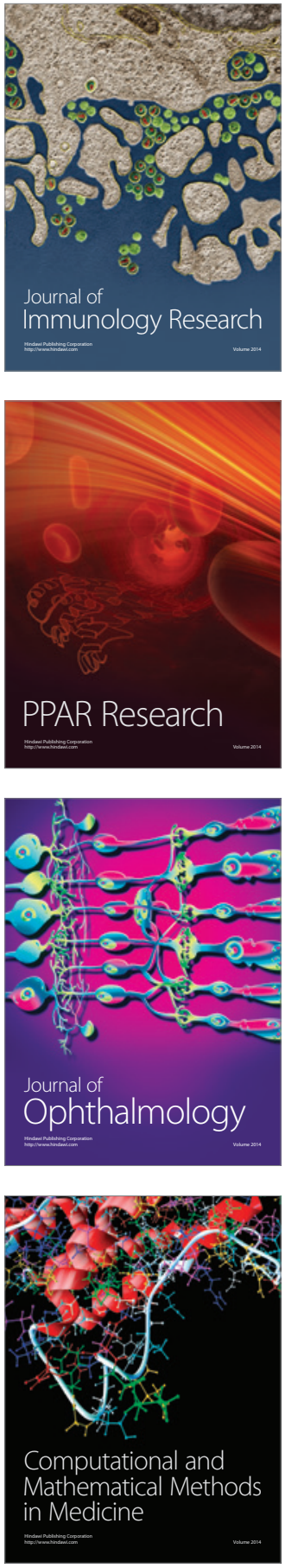

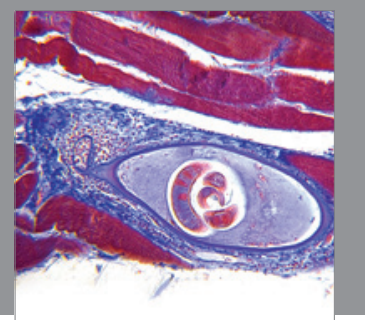

Gastroenterology

Research and Practice
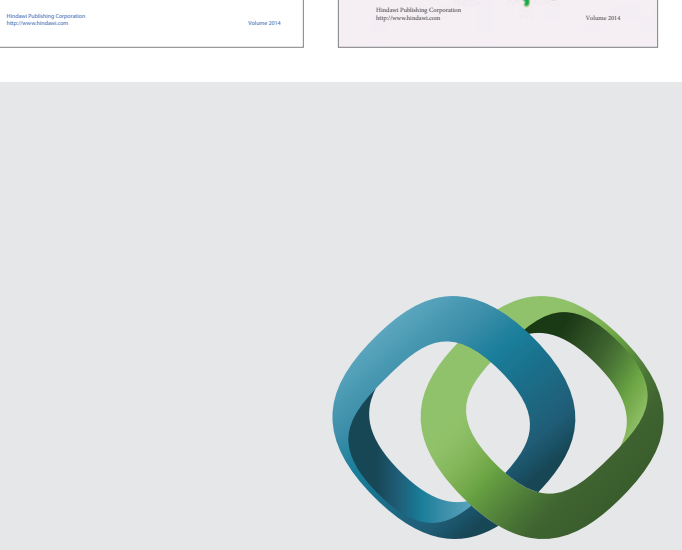

\section{Hindawi}

Submit your manuscripts at

http://www.hindawi.com
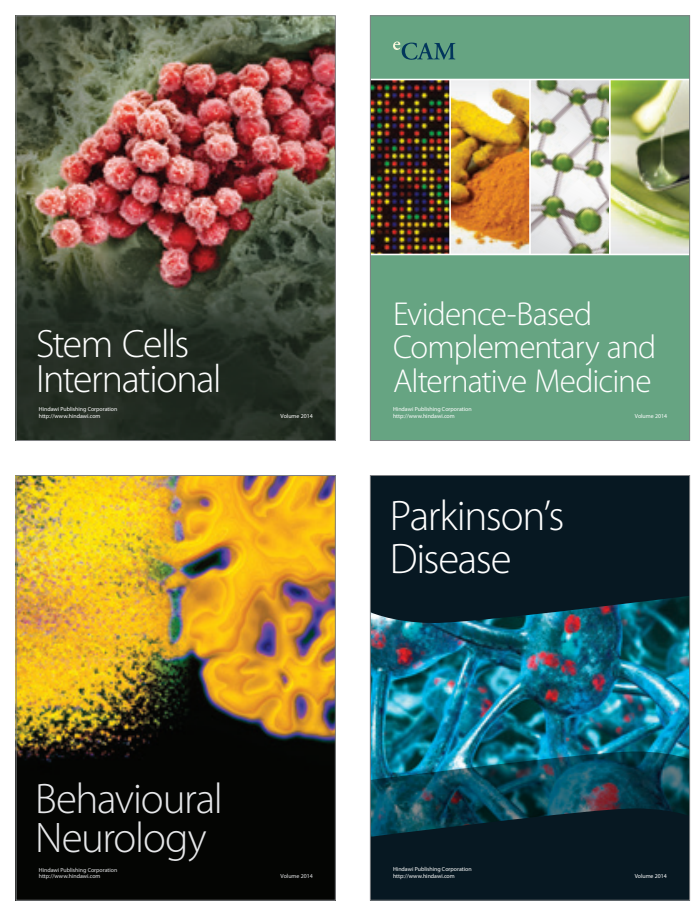

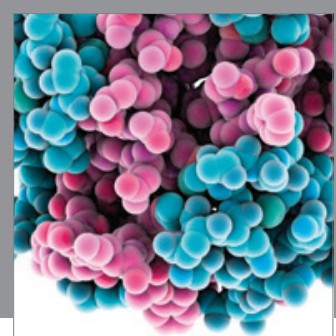

Journal of
Diabetes Research

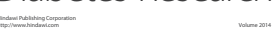

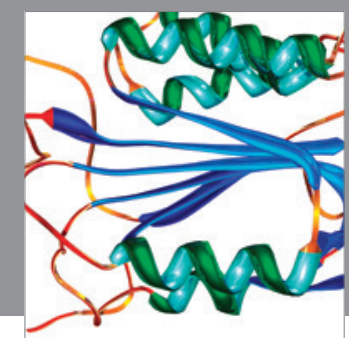

Disease Markers
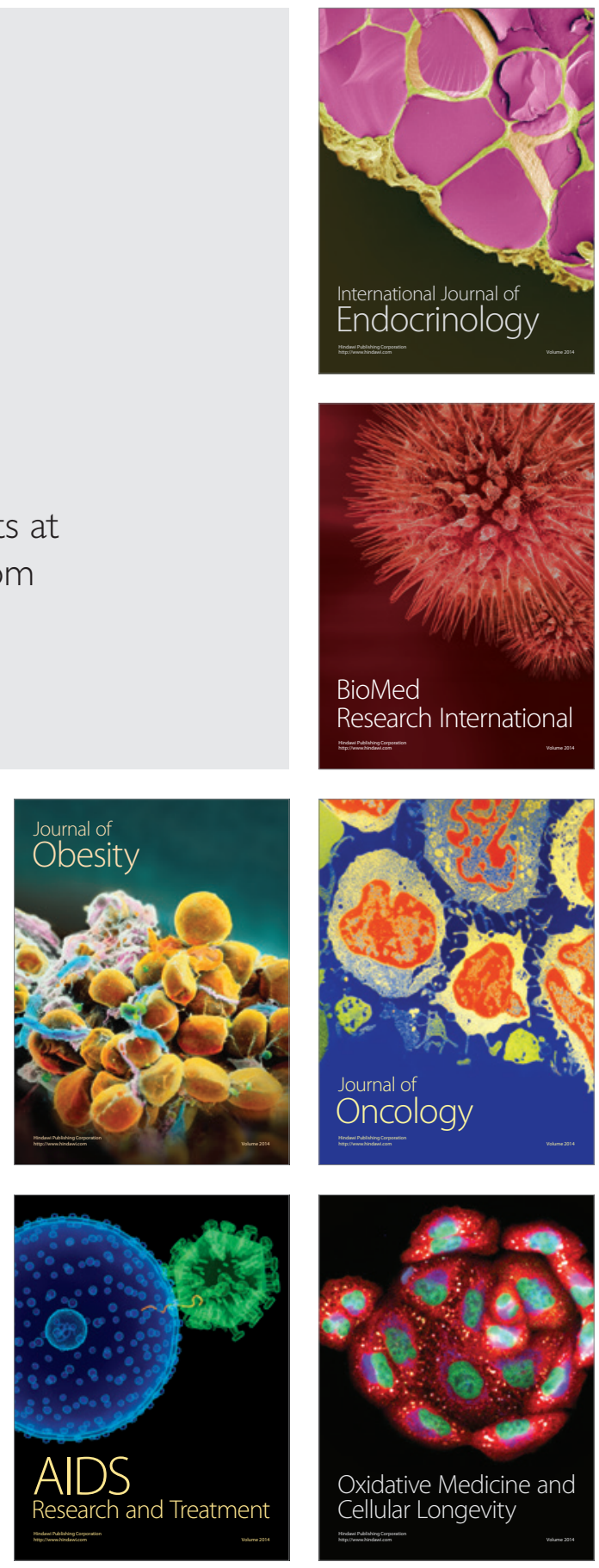